\title{
Caracterización de fincas productoras de palto (Persea americana Mill.) y mandarina (Citrus spp.) en Cañete, Lima, Perú.
}

\author{
Characterization of avocado (Persea americana Mill.) and tangerine (Citrus spp.) \\ farms in Cañete, Lima, Peru.
}

Rubén Collantes González ${ }^{1}$, Alexander Rodríguez Berrio ${ }^{1}$ y Manuel Canto Sáenz ${ }^{1}$

\section{RESUMEN}

Este estudio caracteriza las fincas cultivadas con palto y mandarina en Cañete, Lima (Perú). De los 55 miembros de la Asociación de Agricultores de Cañete dedicados a dichos frutales, se seleccionó una muestra $(n=48)$, para realizar una encuesta sobre aspectos técnicos, ambientales y socio-económicos. Los resultados obtenidos indicaron que las fincas son de extensión variable, manejo intensivo, dependencia media a alta de insumos externos, siendo el $81.25 \%$ de los productores orientados a la agroexportación, lo cual exige implementar Buenas Prácticas Agrícolas (BPA) y acatar disposiciones del Servicio Nacional de Sanidad Agraria (SENASA), sobre control de moscas de la fruta, en especial Ceratitis capitata. Adicionalmente, el $4.17 \%$ de los agricultores dedica pequeñas extensiones a cultivos básicos y un 14.58\% cría animales para autoconsumo. El análisis de conglomerado (clúster), por el método de Ward a una distancia euclidiana cuadrada fijada a ocho, conformó cinco grupos, de los cuales el primero comprende las fincas $3,5,33,41,47$ y 48 , pertenecientes a productores de mandarina con nivel técnico, servicios completos, área cultivada superior a 21 ha, costo productivo superior a PEN 15 000.00, rendimiento superior a 45 t.ha ${ }^{-1}$ y exportan.

Palabras clave: palta; mandarina; agroexportación; costa peruana.

\begin{abstract}
This research was conducted in Cañete, Lima (Peru), in order to characterize avocado and tangerine farms. From the 55 members of the Farmers Association of Cañete, dedicated to these crops, a sample $(n=48)$ was selected to conduct a survey about technical, environmental and socio-economic aspects. The results indicated that the farms are of variable extension, intensive management, average high dependence on external inputs, being $81.25 \%$ of the farmers oriented to agro-exports, which demands the implementation of Good Agricultural Practices (GAP) and fulfill provisions of the National Service of Agrarian Health (SENASA) on the control of fruit flies, especially Ceratitis capitata. Additionally, $4.17 \%$ of farmers dedicated small extensions to basic crops and breeding animals $14.58 \%$ for consumption. The cluster analysis by the Ward method with a square Euclidean distance set to eight formed five groups. The first group included farms 3, 5, 33, 41, 47 and 48, which belong to tangerine produc-
\end{abstract}

${ }^{1}$ Universidad Nacional Agraria La Molina. Lima, Perú. 
ers-exporters with instruction level as technicians, complete services, more than 21 ha of crops, productive costs superior than PEN 15 000.00, yields exceeding $45 \mathrm{t} \mathrm{ha}^{-1}$.

Keywords: avocado; tangerine; agro exports; Peruvian coast.

\section{ICHIKLLACHAW}

Kay uryaqa alli rikapan imanaw paltuwan mandarina murukaayanqanta Kañitichaw, Lima, Piru. Pitsqa chukna pitsqa quchukashqa runakunash tsay mikuykunata muruyan Kañitichaw. Tsaypita akrakashqa muestrapaq ( $\mathrm{n}=48$ ), paykunata inkuwistakashqa kimantinpaq, tikniku, ambiyintal, niykur susiyuikunumikupaq. Paykunapa yaskiyninkuna willakun chakrakunaqa manash tsaynawllatsu kayan, wakin kaqchaw atska uryayan, dipindiyan wak kaq insumukunapita, niykur $81.25 \%$ kusichankuna huk suyukunaman apayaananpaq, tsaypaq alli chakrachaw uryayta wanayan (ACUW), SENASA ninqnta allimpa ruray, frutakuna chuspikunata kuntrulayananpaq, Ceratitis capitata nishqantanaaqa. Hananman, 4.17\% chakra runakuna ichik murupakuyta rurayan, niykur $14.58 \%$ ashmakunata waatakuayn kikinkunalla mikuyaananpaq. Alli ñawipaykuna (klustir), Warpa mitudunwan patsata iwclidiyana cuadradata churayan puwaqta, aylluyan pitsqa quchukunata, tsaypita, nawpakaqkuna tikutsiyan 3, 5, 33, 41, 47 niykur 48, mandarina chawpi niwilwan muruqkunata, llapan ruraykuqkunata, chakrakuna murukashqata 21 ha, hananpa, pruduksiyunpa chanin PEN 15000.00 hananpa, alli rindimiyintuyuq 45 t.ha ${ }^{-1}$ hananpa, niykur wak suyukunapaq apatsiyan.

Pushaq shimikuna: palta; mandarina, agruikspurtasiyun, achachaq prupa markan.

\section{INTRODUCCIÓN}

El palto (aguacate) y el mandarino son frutales tropicales cuyos frutos brindan aportes nutricionales y nutracéuticos, siendo por ello importantes para consumo fresco y la industria (alimentos, medicinas, cosméticos, entre otros). Representan ofertas exportables atractivas, por lo cual su cultivo en la costa peruana están en expansión, siendo el Valle de Cañete, situado al sur de la provincia de Lima, una zona productiva con 1555 ha y 1075 ha de mandarina y palto, respectivamente (MINAGRI, 2014).

Durante el siglo pasado, Cañete tuvo una evolución interesante en el sector agrario, llegando a ser una zona productora importante de algodón, el cual sirvió para desarrollar el Manejo Integrado de Plagas (MIP) en Perú. Sin embargo, la reforma agraria ocasionó que las haciendas pasaran a conformar cooperativas y, posteriormente, parcelas, descontinuándose todo avance y retomándose la agricultura convencional (Herrera, 2010).

La dependencia de agroquímicos afecta el equilibrio natural e interfiere con los servicios ambientales, atentando contra la sustentabilidad del agroecosistema. Existen limitantes conceptuales y materiales para tomar decisiones, requiriéndose herramientas de análisis prácticas y aplicadas (Carson, 1962; Sarandon y Flores, 2009; Altieri y Nicholls, 2010).

La caracterización es una fase básica, descriptiva, cualitativa (apoyada en antecedentes y datos organizados), para identificar y caracterizar componentes, acontecimientos, actores, procesos y contexto de una experiencia o hecho y posteriormente definirlo; siendo vital en la agricultura para determinar variables que permitan distinguir zonas, 
predios o agroecosistemas de interés (Malagón y Praguer, 2001; Strauss y Corbin, 2002; Bonilla et al., 2009; Sánchez-Upegui, 2011).

El objetivo del presente estudio ha sido caracterizar las fincas productoras de palto y mandarina en el Valle de Cañete, Lima, Perú.

\section{MATERIALES Y MÉTODOS}

El estudio se desarrolló en la provincia de Cañete, Lima-Perú, con una superficie de $4580 \mathrm{~km}^{2}$. Limita al norte con las provincias de Lima y Huarochirí, al este con la provincia de Yauyos, al sur con el departamento de Ica y al oeste con el Océano Pacífico. Se ubica a $13^{\circ} 04^{\prime} 42^{\prime \prime}$ LS $76^{\circ} 23^{\prime} 02^{\prime \prime}$ LO, con una altitud de 3 - 819 msnm., clima subtropical seco, temperatura promedio de $19,7^{\circ} \mathrm{C}$, siendo $28^{\circ} \mathrm{C}$ en verano y $14-20^{\circ}$ $\mathrm{C}$ en invierno, con escasa precipitación de $26 \mathrm{~mm}$. año ${ }^{-1}$ y humedad relativa entre 81 $-87 \%$ (INDECI, 2002).

Como población objetivo, se seleccionó fincas productoras de palto y mandarina inscritas en la Asociación de Agricultores de Cañete, obteniéndose mediante el método de proporciones recomendado por Julca et al. (2009):

$$
n=\frac{\frac{4 P Q}{d^{2}}}{\frac{\frac{4 P Q}{d^{2}}-1}{N}+1}
$$

Donde:

$$
\begin{aligned}
& \mathrm{n}: \text { tamaño de muestra } \\
& \mathrm{N}: \text { población objetivo (Universo) } \\
& \mathrm{P}: \text { probabilidad de acierto }(0,5) \\
& \mathrm{Q}: \text { probabilidad de error }(0,5) \\
& \mathrm{d}: \text { error }(5 \text { por ciento) }
\end{aligned}
$$

De un total de 55 fincas, se obtuvo una muestra irrestricta aleatoria [ $\mathrm{n}=48$ ]. Cada finca visitada fue georreferenciada con GPS (figura 1), para facilitar la programación de trabajo (100 horas).

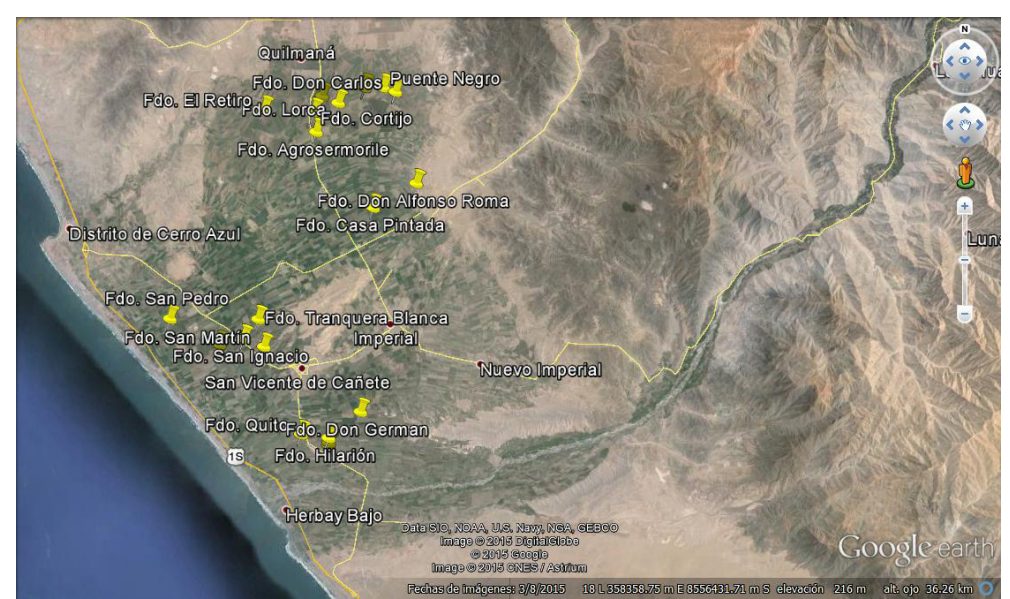

Figura 1. Georreferenciación de fincas visitadas en el Valle de Cañete, Lima-PE 
La información de las fincas situadas en los distritos de San Vicente, San Luis, Quilmaná, Imperial y Nuevo Imperial, se obtuvo mediante una encuesta estructurada considerando las siguientes dimensiones:

Social: sexo del encuestado, edad, nivel de instrucción, número de personas con las que vive, servicios disponibles, acceso a salud y educación, lugar de residencia asociatividad.

Económica: área de la finca, título de propiedad, crianza de animales, cultivo principal, cultivo secundario, otras actividades económicas, área cultivada (ha), costo productivo (PEN.ha ${ }^{-1}$, tasa de cambio al momento de la evaluación fue 1 PEN = 0.3125 USD), trabajadores requeridos, rendimiento $\left(\mathrm{t} . \mathrm{ha}^{-1}\right)$, calidad, lugar y precio de venta y ganancia.

Técnico-ambiental: tipo de cultivos (monocultivo o diversificados), manejo de la finca (convencional o transición), insumos, procesamiento de producto, capacitación recibida, responsable de la capacitación y temas de interés en los cuales desean capacitarse.

Finalmente, se realizó un análisis de conglomerado (clúster), por el método de Ward y distancia euclidiana cuadrada fijada a ocho, siendo las variables consideradas:

Sociales: nivel de instrucción del agricultor, servicios disponibles (agua, luz, teléfono, centros de salud, entre otros) y participación en algún tipo de asociación.

Económicas: crianza de animales, cultivo principal, área cultivada, costo del cultivo principal, rendimiento $\left(\mathrm{t}^{\mathrm{h}} \mathrm{h}^{-1}\right)$ y si exportan.

Técnico-Ambiental: tipo de agricultura (convencional, ecológica o en transición).

\section{RESULTADOS}

1. Dimensión social de las fincas visitadas

El 87.5\% de los agricultores son del sexo masculino (figura 2); con edades distribuidas en los rangos de 25-40 (22), 41-55 (10) y mayores a 56 años (10) (figura 3); y nivel de instrucción mayormente técnico (25) (figura 4). De las mujeres encuestadas (figura 2), cuatro tienen edades comprendidas entre 41-55 años (figura 3), y tres tienen formación superior (figura 4).

El número de personas por vivienda (figura 5), tuvo una moda de cuatro y excepcionalmente un caso con 10 . Adicionalmente, 32 viviendas $(66.67 \%)$, cuentan con todos los servicios básicos (figura 6) y el 50\% de las viviendas están ubicadas en la ciudad (figura 7).

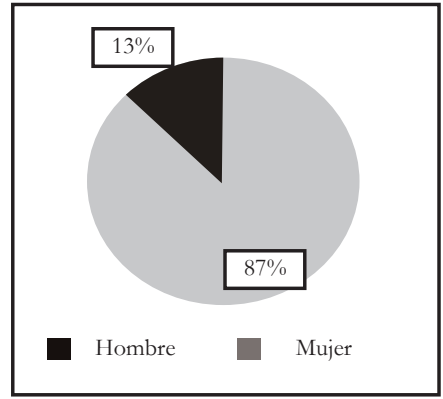

Figura 2. Sexo de los encuestados (\%)

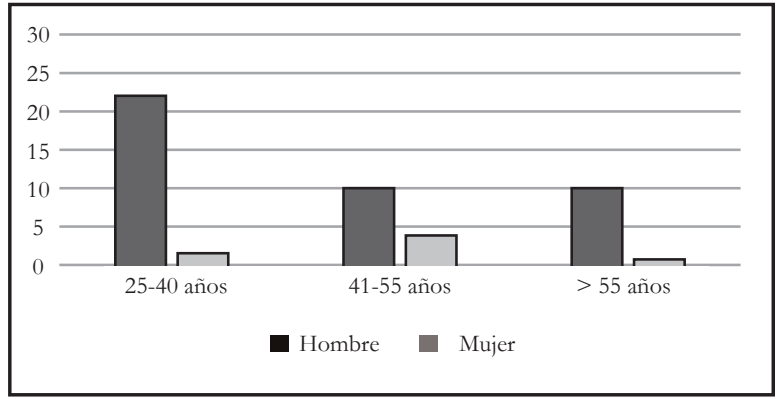

Figura 3. Distribución de edades según el sexo 


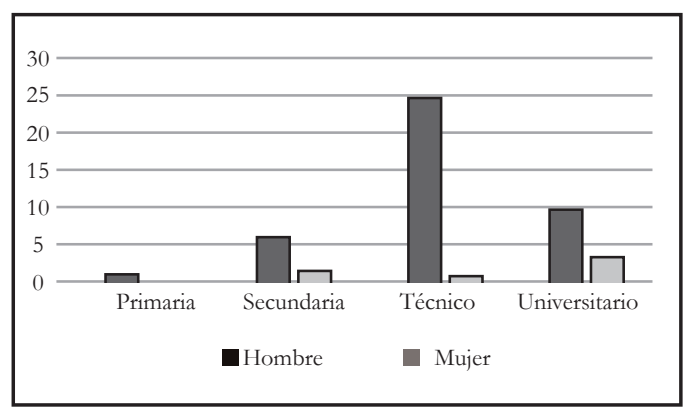

Figura 4. Nivel de instrucción según el sexo

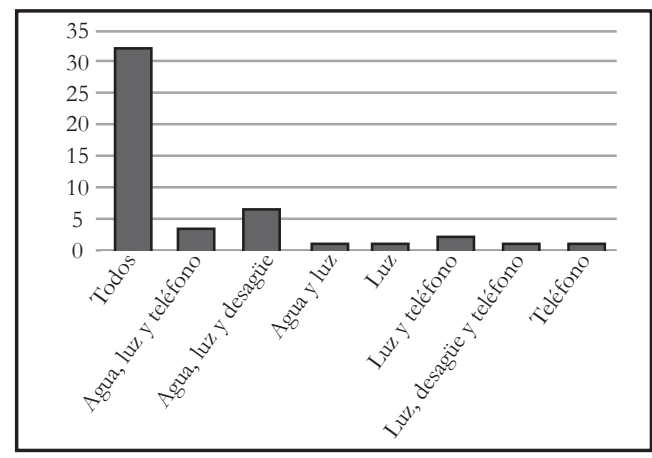

Figura 6. Acceso a servicios básicos

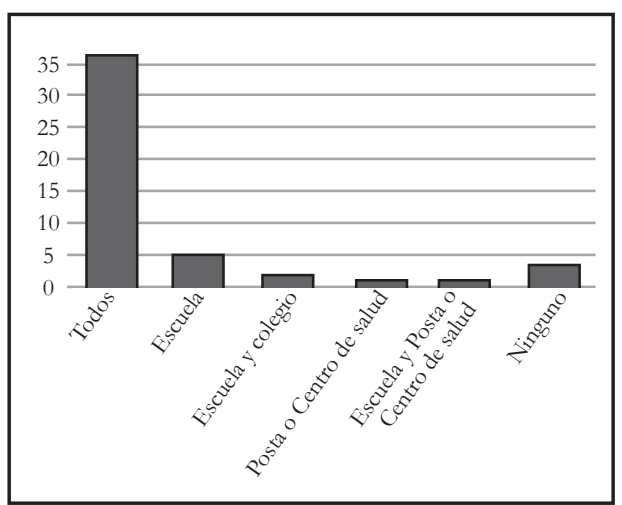

Figura 8. Acceso a salud y educación

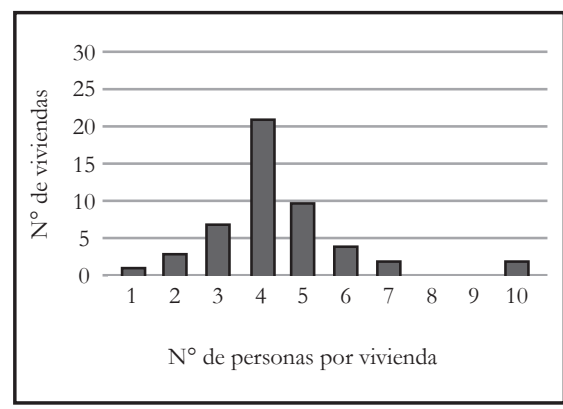

Figura 5. Número de personas por vivienda

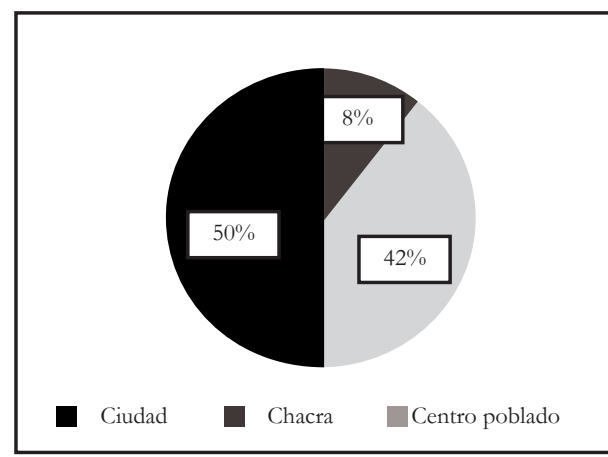

Figura 7. Ubicación de la residencia (\%)

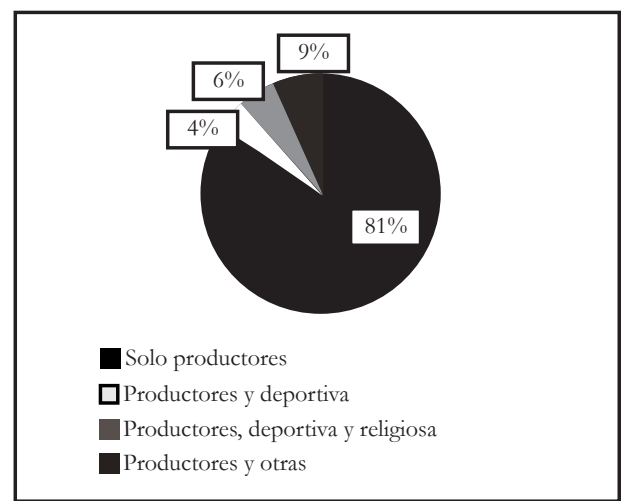

Figura 9. Participación en asociaciones (\%)

El 75\% de los encuestados (36), cuentan con escuelas, colegios y centros médicos próximos a sus viviendas; mientras que el 18.75\% (9), no cuentan con todos los servicios (figura 8); y el 6.25\% (3), no cuenta con ningún servicio. En cuanto a la asociatividad (figura 9), todos los encuestados pertenecen a la Asociación de Agricultores y algunos participan en otros colectivos.

\section{Dimensión económica de las fincas visitadas}

De 1789.4 ha recorridas se cultivan 1669.9 ha (93.32\%) (figura 10) y el $91.67 \%$ de las fincas están tituladas (figura 11). El 42\% son agricultores medianos (21-50 ha), 27\% medianos-pequeños, $23 \%$ medianos-grandes y finalmente $8 \%$ pequeños (figura 12). 
En cuanto a la crianza animal (figura 13), 4\% posee aves de corral, 6\% cría ovinos y/o caprinos, $4 \%$ cría cuyes y solo $2 \%$ tiene animales menores y mayores. En lo referido a cultivos (figura 14), 24 fincas tienen como cultivo principal el mandarino y 19 el palto; cinco tienen por cultivo secundario el mandarino y cuatro el palto. Sobre ingresos alternativos (figura 15), 4\% de los agricultores participan en el comercio y 10\% desarrollan otras actividades.

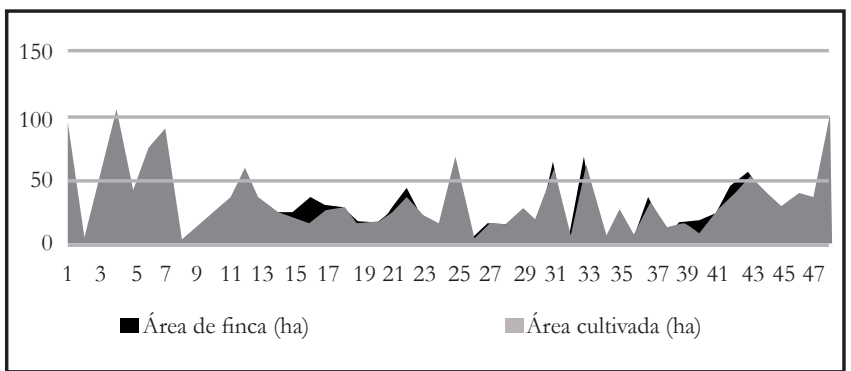

Figura 10. Área de la finca (ha) vs. Área cultivada (ha)

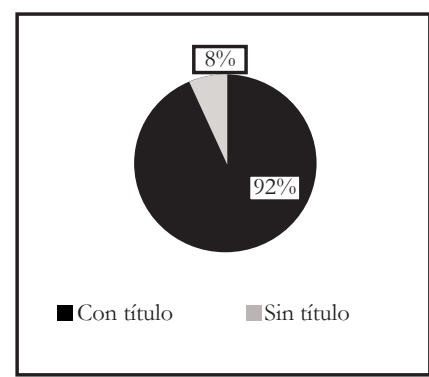

Figura 11. Tenencia de título $(\%)$

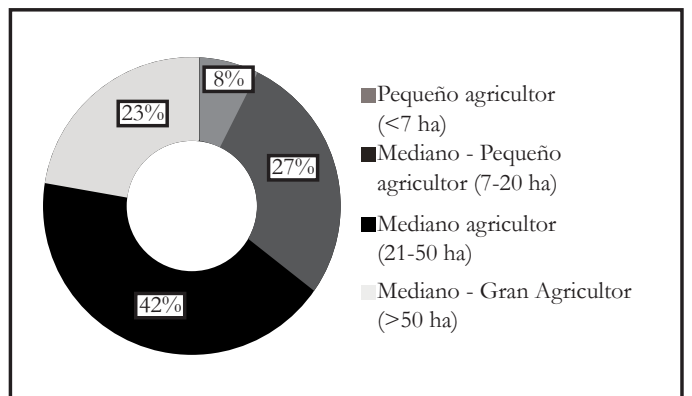

Figura 12. Agricultores según área cultivada (ha)

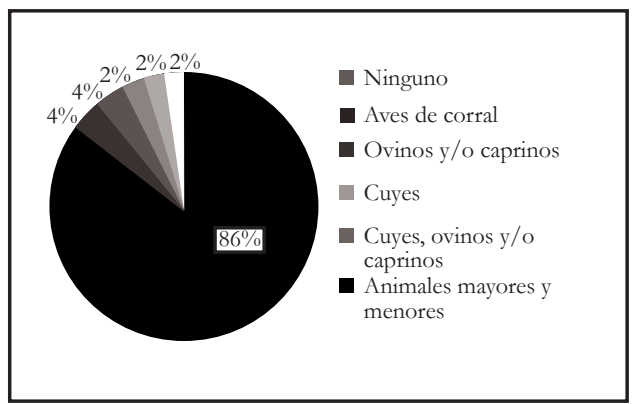

Figura 13. Crianza animal (\%)

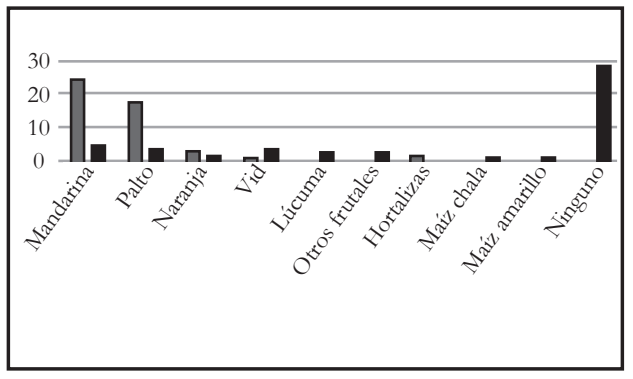

Figura 14. Cultivos principal y secundario

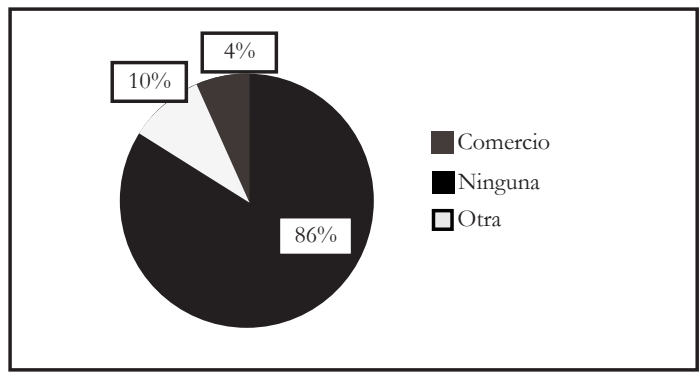

Figura 15. Actividades económicas alternativas

El costo de producción (figura 16), para el cultivo principal fue variable, entre PEN 700.00.ha h $^{-1}$ y PEN 36 000.00.ha , $^{-1}$ con promedio de PEN 17 120.00.ha . $^{-1}$ y moda PEN 15 000.00.ha ${ }^{-1}$; mientras que para el cultivo secundario fue desde PEN 700.00.ha ${ }^{-1}$ hasta PEN 30 000.00.ha , $^{-1}$ promedio de PEN 5700.00.ha y $^{-1}$ moda PEN 0.00.ha ( $^{-1}$ (28 fincas sin cultivos secundarios). El número de trabajadores varió de uno a 70 por finca, con un promedio de 16 y moda de ocho. Esto estuvo parcialmente correlacionado con la inversión por finca $\left(\mathrm{R}^{2}=0.44\right)$ (figura 17). 


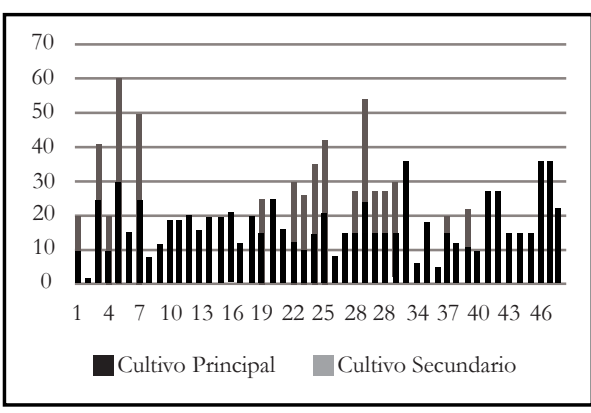

Figura 16. Costo productivo (miles de PEN.ha ${ }^{-1}$ )

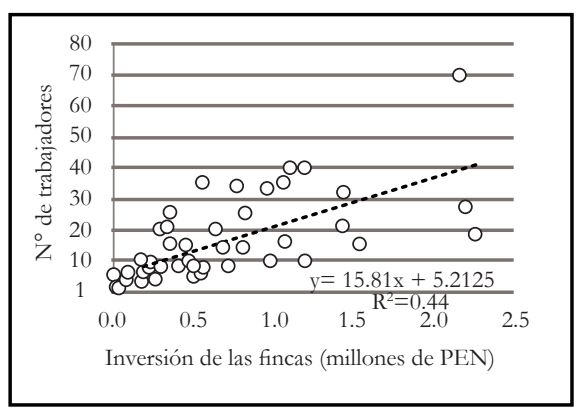

Figura 17. Trabajadores vs. Inversión

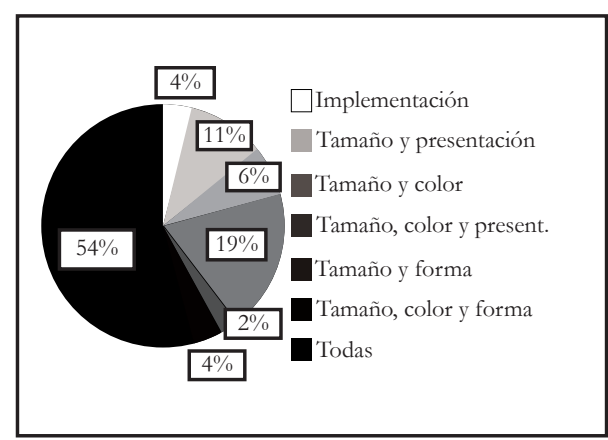

Figura 18. Rendimiento $\left(\right.$ t.ha $\left.^{-1}\right)$, por finca visitada Figura 19. Determinación de la calidad

Al comparar los rendimientos obtenidos (figura 18), la mandarina registró los mayores

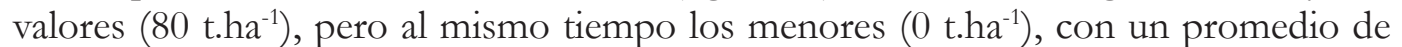
41.27 t.ha ${ }^{-1}$; mientras que el palto varió entre 5 t.ha ${ }^{-1}$ y 22 t.ha ${ }^{-1}$, con un promedio 13.74 t.ha ${ }^{-1}$. En cuanto a la determinación de la calidad de frutos para comercializar (figura 19), el $96 \%$ de los encuestados consideró el tamaño como el atributo principal, sumado a otros complementarios.

Respecto a los mercados de destino (figura 20), el 81\% de los agricultores exportan parte de sus cosechas; mientras que un 17\% opta por venderlo en la chacra y solo un $2 \%$ lo comercializa en el mercado local. En cuanto al precio de venta de la fruta (figura 21), durante la campaña 2014-2015 fue variable, siendo el valor mínimo registrado PEN 0.80. $\mathrm{kg}^{-1}$ y el máximo hasta PEN 5.00. $\mathrm{kg}^{-1}$, con un promedio de PEN 2.27. $\mathrm{kg}^{-1} \mathrm{y}$ una moda de PEN 2.00. $\mathrm{kg}^{-1}$.

Sobre las ganancias generadas, en una finca cuyo cultivo principal es la mandarina se obtuvo hasta PEN 290 500.00.ha ${ }^{-1}$ de utilidad; mientras que en palto la máxima ganancia obtenida fue de PEN 54 400.00.ha ${ }^{-1}$. En la figura 22, se presenta las ganancias totales por finca visitada.

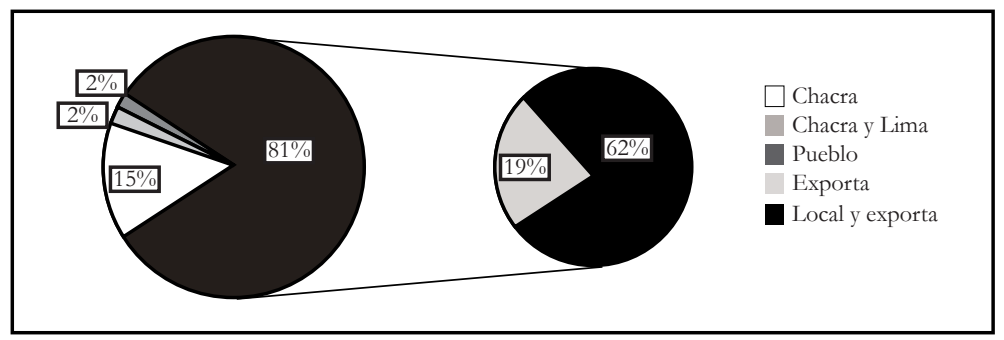

Figura 20. Destinos de comercialización, campaña 2014-2015 


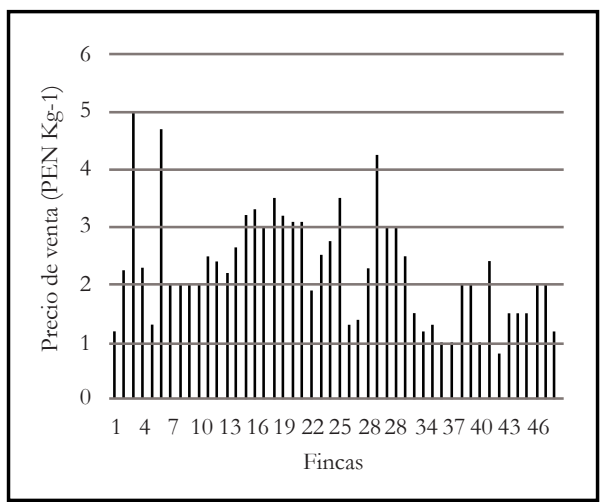

Figura 21. Precio de venta de los productos

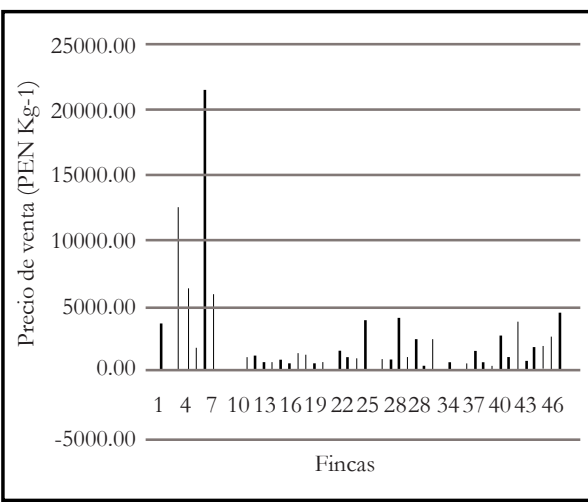

Figura 22. Ganancia por finca (miles de PEN)

3. Dimensión técnico-ambiental de las fincas visitadas

Predominó el monocultivo (58\%) (figura 23) y el manejo convencional con Buenas Prácticas Agrícolas (95.83\%). Los insumos más empleados fueron los agroquímicos y plantas injertadas y certificadas; mientras que un 50\% de los agricultores encuestados utiliza abonos orgánicos. En cuanto al procesamiento del producto, solo el 14.58\% lo procesa.

Respecto a las capacitaciones, solo un agricultor señaló no haber recibido capacitaciones; mientras que el $97.92 \%$ de los agricultores afirmaron haber sido capacitados sobre primeros auxilios y recojo y entierro de frutas, por parte del Cuerpo de Bomberos y el SENASA, respectivamente. Adicionalmente, 32 agricultores han sido capacitados por la Asociación de Agricultores de Cañete y/o el Estado, mientras que cuatro han sido capacitados por alguna ONG y 11 mediante asesoría particular (figura 24). Los temas principales de capacitaciones recibidas son fertilización $(97.72 \%)$, control de plagas (45.83\%), comercialización (45.83\%), entre otros $(2.08 \%)$; siendo esta misma tendencia el interés de los agricultores para futuras capacitaciones.

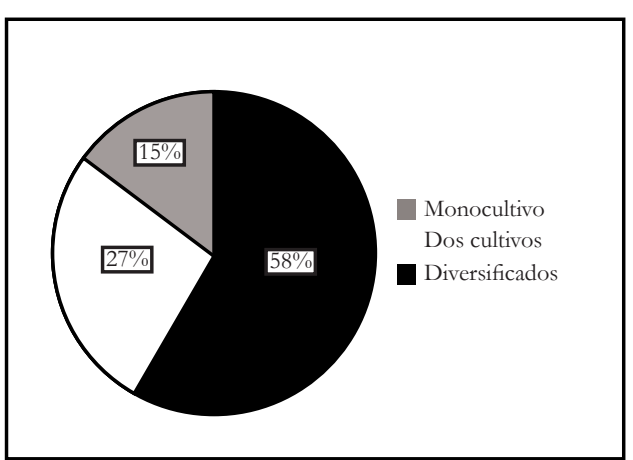

Figura 23. Tipos de cultivo desarrollados en Cañete

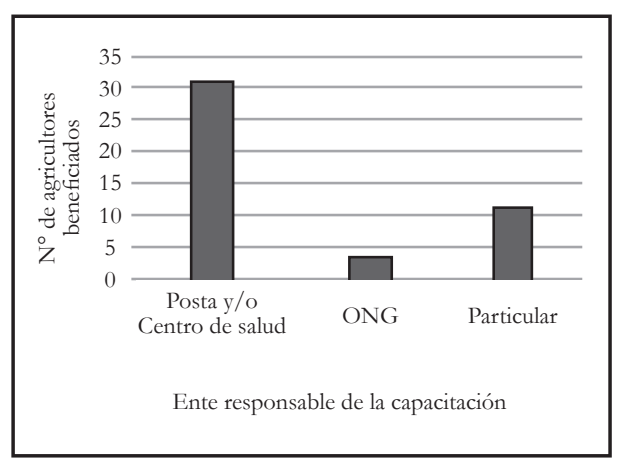

Figura 24. Responsable de capacitación

4. Análisis de conglomerados (Clúster), por el Método de Ward

A una distancia euclidiana cuadrada fijada a 8 (figura 25), se conformó cinco grupos, de los cuales el primero comprende las fincas 3, 5, 33, 41, 47 y 48, pertenecientes a productores de mandarina con nivel técnico, servicios completos, cultivo superior a 21 ha, costo productivo superior a S/. 15000.00 , rendimiento superior a 45 t.ha $^{-1} \mathrm{y}$ además exportan. 


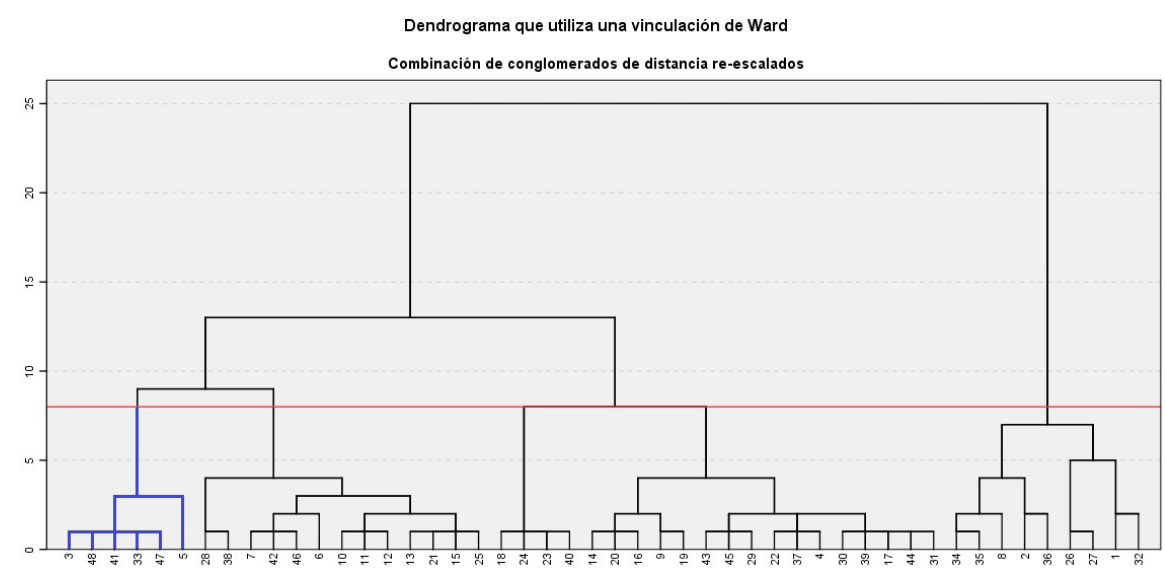

Figura 25. Análisis de Conglomerados (Clúster), fincas de palto y mandarina en Cañete

\section{DISCUSIÓN}

Si bien el mayor porcentaje de agricultores encuestados fueron hombres, se observó durante la labor en campo mujeres desarrollando diversas faenas, lo cual indica que los cultivos agroexportables como el palto y la mandarina contribuyen al empoderamiento familiar, brindando oportunidad igualitaria de generar ingresos mediante el trabajo, concordando con lo propuesto por FIDA (2012).

En cuanto a los servicios, todos los encuestados cuentan con teléfono celular y pese a poder obtener teléfono fijo, desestiman el mismo ya que consideran prioritaria la rapidez para atender llamadas mientras trabajan en campo. Si bien no todos los agricultores tienen cercanía a centros médicos, se observó que las fincas cuentan con botiquines de primeros auxilios, tienen señalizaciones para la disposición de desechos y envases de plaguicidas y exigen a los trabajadores el uso del equipo de protección personal completo, en cumplimiento con el Reglamento de Seguridad y Salud en el Trabajo (DS No 009-2005-TR) y el Manual de Salud Ocupacional elaborado por DIGESA (2005).

Adicionalmente, en los fundos visitados se observó la presencia de cintas azules del SENASA, lo cual indica el interés por parte de los agricultores en preservar áreas libres de plagas cuarentenarias, favoreciendo la agroexportación y el desarrollo del PROSCV-01 de SENASA (2006).

Inversiones elevadas no necesariamente han generado mayores utilidades para la campaña 2014-2015, ya que en varios fundos han optado por instalar sistemas de riego y renovar plantas. Plantaciones antiguas de mandarina Clementina han sido sustituidas por cultivares como W. Murcot, Satsuma Okitsu, Tango y Primosole, utilizando como nuevo patrón Citrumelo, debido a que el mandarino Cleopatra induce la producción tardía por su desarrollo radicular, así como un tamaño reducido de frutos en plantas adultas, corroborando lo expuesto por Orduz-Rodríguez y Mateus (2012). En nuevas plantaciones de palto Hass han utilizado Zutano como polinizante y patrón, el cual es más vigoroso, más tolerante a sales y enfermedades como la pudrición radicular (Phytophthora cinnamomi), esto último probado por Apaza et al. (2015), en la Irrigación Chavimochic. Esto explica el por qué en plantaciones nuevas no están considerando usar Topa Topa como patrón. 
El análisis clúster ha conformado grupos de fincas semejantes entre sí, lo cual facilitará a futuro desarrollar evaluaciones más detalladas optimizando recursos, lo cual concuerda plenamente con lo sugerido por Malagón y Praguer (2001), Strauss y Corbin (2002) y Sánchez-Upegui (2011).

\section{CONCLUSIONES}

Se caracterizó las fincas productoras de palto y mandarina en Cañete, las cuales socialmente representan alternativas de desarrollo y empoderamiento igualitario en cuestión de géneros. Económicamente, las fincas visitadas requieren una inversión promedio de PEN 17 120.00.ha-1 ${ }^{-1}$, siendo más rentable el cultivo de mandarina al superar hasta tres veces en promedio los rendimientos obtenidos en palta y cinco veces las utilidades generadas por hectárea cultivada.

Ambientalmente, pese a las buenas prácticas adoptadas en las fincas visitadas, se requiere cambiar el enfoque productivo de manejo convencional a Manejo Integrado del Agroecosistema.

El análisis de conglomerado (clúster), por el método de Ward a una distancia euclidiana cuadrada fijada a ocho, conformó cinco grupos, de los cuales destacó el primero que comprende las fincas 3, 5, 33, 41, 47 y 48, pertenecientes a productores de mandarina con nivel técnico, servicios completos, área cultivada superior a 21 ha, costo productivo superior a PEN 15 000.00, rendimiento superior a 45 t.ha ${ }^{-1}$ y exportan.

\section{AGRADECIMIENTOS}

Al Museo de Entomología Dr. Klaus Raven B. de la UNALM, al IRD-Costa, UNALM y al Instituto Valle Grande, por el apoyo logístico. A Percy Peralta y al Ing. Felizardo Fabián, por el apoyo durante la fase de campo. Finalmente, a los agricultores por su gentil colaboración.

\section{REFERENCIAS BIBLIOGRÁFICAS}

Altieri, Miguel y Nicholls, Clara. 2010. Diseños Agroecológicos para Incrementarla Biodiversidad de Entomofauna Benéfica en Agroecosistemas. $1^{\text {ra }}$ ed. Medellín: Sociedad Científica Latinoamericana de Agroecología (SOCLA).

Apaza, Walter y otros. 2015. «Comportamiento de los cinco patrones de palto más utilizados en la irrigacion de Chavimochic a la pudricion radicular ocasionada por Phytophthora cinnamomi». Poster presentado en el VIII Congreso Mundial de la Palta. Lima: Libro de Resúmenes. 51.

Bonilla, Elssy; Hurtado, Jimena y Jaramillo, Christian. 2009. Lainvestigación: Aproximaciones a la construcción del conocimiento cientifico. Bogotá: Alfaomega.

Carson, Rachel. 1962. Silent Spring. Edición del 50 Aniversario. Mariner Books: US.

DIGESA (Dirección General de Salud Ambiental). 2005. Manual de Salud Ocupacional. Lima: Ministerio de Salud. Dirección Ejecutiva de Salud Ocupacional. 
FIDA (Fondo Internacional de Desarrollo Agrícola). 2012. Igualdad de género y empoderamiento de la mujer. Roma: IT.

Herrera, Juan. 2010. Primera experiencia a nivel mundial del Manejo Integrado de Plagas: el caso del algodonero en el Perú. Lima: Rev. Per. Ent.

INDECI (Instituto Nacional de Defensa Civil). 2002. Mapa de peligros, Plan de usos del suelo y Propuesta de medidas de mitigación de los efectos producidos por los desastres naturales de las ciudades de la Provincia de Cañete. Lima: Programa de Ciudades Sostenibles, Primera Etapa en colaboración con Universidad Nacional San Luis Gonzaga de Ica.

Julca, Alberto y otros. 2006. Selección de fuentes naturales para la fertilización de café en el marco de una agricultura orgánica. Lima: Línea base de Proyecto financiado por INCAGRO, en alianza con la UNALM, la FDA, la JNC y el INIEA.

Malagón, Ricardo y Praguer, Martín. 2001. El enfoque de sistemas: Una opción para el análisis de las unidades de producción agrícola. Bogotá: Universidad Nacional de Colombia.

MINAGRI (Ministerio de Agricultura y Riego). 2014. Consolidado de la Información Agraria de la Provincia de Cañete. Lima: Oficina de Información Agraria, Agencia Agraria de Cañete.

MTPE (Ministerio de Trabajo y Promoción del Empleo). 2005. Reglamento de Seguridad y Salud en el Trabajo. Decreto Supremo No 009-2005-TR. 21.

Orduz-Rodríguez, Javier y Mateus, Diana. 2012. «Generalidades de los cítricos y recomendaciones agronómicas para su cultivo en Colombia». Cap. 2. Citricos: Cultivo, poscosecha e industrialización. Serie Lasallista Investigación y Ciencia. 49-88.

Sánchez-Upegui, Alexander. 2011. Manual de Redacción Académica e Investigativa: Cómo escribir, evaluar y publicar artículos. Medellín: Católica del Norte Fundación Universitaria.

Sarandón, Santiago y Flores, Claudia. 2009. «Evaluación de la sustentabilidad en agroecosistemas: Una propuesta metodológica». Agroecología No 4. 19-28.

SENASA (Servicio Nacional de Sanidad Agraria). 2006. Procedimiento: Establecimiento, Mantenimiento y Verificación de Áreas Libres de Plagas de Moscas de la Fruta en el Perú. Lima: Dirección de Sanidad Vegetal, Subdirección de Cuarentena Vegetal. PROSCV-01.

Strauss, Anselm y Corbin, Juliet. 2002. Bases de la investigación cualitativa: Técnicas y procedimientos para desarrollar la teoría fundamentada. Medellín: Universidad de Antioquia.

Fecha de recepción: 07 de febrero de 2015

Fecha de aceptación: 10 de abril de 2015 
||Rubén Collantes, Alexander Rodríguez, Manuel Canto

\section{Correspondencia}

Rubén Collantes González

rdcg31@hotmail.com 